\title{
Vehicle-to-Pedestrian Channel Characterization: Wideband Measurement Campaign and First Results
}

\author{
Ibrahim Rashdan, Fabian de Ponte Müller, Wei Wang, Martin Schmidhammer and Stephan Sand \\ German Aerospace Center (DLR) \\ Institute of Communications and Navigation, 82234 Oberpfaffenhofen, Germany \\ \{Ibrahim.Rashdan | Fabian.PonteMueller | Wei.Wang | Martin.Schmidhammer, | Stephan.Sand\}@dlr.de
}

\begin{abstract}
Thousands of fatalities among pedestrians are caused every year by traffic accidents. Vehicle-to-pedestrian (V2P) communication promises to prevent accidents by enabling collision avoidance application. To develop and test a V2P communications system, accurate knowledge of the propagation channel is essential. However, only limited analysis of V2P channel have been reported in the literature. To fill this gap, the German Aerospace Center conducted an extensive channel sounding measurements campaign in a controlled environment. The measurements were performed at $5.2 \mathrm{GHz}$ with a bandwidth of $120 \mathrm{MHz}$. In parallel to the channel sounding measurements, performance measurements were carried out using ITS-G5 system at $5.9 \mathrm{GHz}$ and with a bandwidth of $10 \mathrm{MHz}$. This paper describes the setup and the scenarios for the two measurements. First results on channel evaluation in different scenarios as well as path loss models are presented.

Index Terms-V2P communication, channel measurements, performance evaluation, IEEE 802.11p, path loss
\end{abstract}

\section{INTRODUCTION}

From a safety perspective, the urban traffic environment is specially challenging due to its high mobility and variety of traffic participants. Cars, motorcycles, pedestrians and bicycles practically share the same space at the same time. Since cyclists and pedestrians are specially exposed to dangerous situations they are categorized as so-called vulnerable road users (VRU). In Europe, VRUs account for $30 \%$ of all road fatalities. Most fatalities, severe and slight injuries to pedestrians and cyclists occur in urban areas [1]. Currently, driver assistance systems and automated vehicles only rely on their own perception sensors to detect and locate other surrounding traffic participants. However, radar sensors, laser-scanners and camera-based systems have critical limitation. They require a direct sight towards the other road users. Additionally, lightbased systems show a low performance under bad weather or lighting conditions. One way to overcome this limitation and to obtain 360 degree of awareness is to use a communication technology to directly exchange information between vehicles and VRUs [2]. Dedicated Short Range Communication (DSRC) in the US or ITS-G5 in Europe are IEEE 802.11pbased technologies for ad-hoc communication. The 3GPP consortium is incorporating V2X capabilities into their LongTerm Evolution (LTE) standard under the name LTE-V2X, which will eventually evolve into the future $5 \mathrm{G}$ standard for mobile network communication.

In order to develop an effective vehicle-to-pedestrian (V2P) communication system, accurate channel models are required.
In recent years, vehicle-to-vehicle (V2V) and vehicle-toinfrastructure (V2I) communication were investigated extensively. The performance of ITS-G5 in various environments has been studied e.g. in [3]. Further, many new channel models for V2V and V2I have been proposed and summarized in surveys like [4] and [5]. The V2P channel model, however, has attracted less attention. Yet, there are crucial differences between the V2P and the V2V or V2I communication channel as for instance:

- Transmit (Tx) and receive ( $\mathrm{Rx})$ antennas in $\mathrm{V} 2 \mathrm{~V}$ have fixed heights. In V2P, however, the antenna at the pedestrian side, which can be integrated within cell phones or wearable devices, may change its orientation and position based on the pedestrian's activity (texting, calling, etc.). This implies that multipath components may have different propagation characteristics than those in $\mathrm{V} 2 \mathrm{~V}$ communication.

- The mobility pattern and velocity differences between vehicles and pedestrians impose different propagation characteristics.

- In V2V communication, signals get obstructed by large objects such as building and other vehicles. In V2P communication, signals will experience additional attenuation caused by the human body, from surrounding pedestrians and from parked vehicles as well as traffic signs and vegetation along the roadside.

Some recent studies tackled the subject of V2P communications. The authors in [6] investigated the communication channel at $5.8 \mathrm{GHz}$ between a stationary pedestrian and a vehicle in an urban environment. Based on the measurement data, the Rice model was found to characterize the observed small scale fading. In [7], real-world tests were reported in an uncontrolled approach to evaluate the IEEE 802.11p and LTE-based V2V communication and WiFi-based V2P communication in terms of packet delivery ratio (PDR) and end-to-end latency. A Wi-Fi-based V2P communication was also studied in [8]. The authors investigated the PDR and the packet inter-reception (PIR) time. In their scenarios, the vehicle was parked on the road and the pedestrian walked away from the vehicle. Authors of [9] also evaluated performance of ITS-G5 for VRU safety services in terms of PDR. However, all these studies and measurements were performed with small bandwidth (maximum $20 \mathrm{MHz}$ ). To the best of our knowledge, there is so far no research group that investigated in detail 
the propagation characteristics of the V2P channel based on channel sounding measurements. Moreover, a dedicated model for V2P communication does not yet exist.

In this paper, we describe a channel measurement campaign that was performed using a channel sounder and an ITSG5 system in a controlled environment. The measurements involve one test vehicle, one pedestrian, and one to six parked cars in different configurations. We show a first evaluation of the channel measurement data by discussing the path loss with each of the configurations and proposing models for its characterization.

\section{PATH LOSS MODELS}

\section{A. Log-distance path loss model}

The log-distance path loss model is used to predict the propagation loss in different environments. The average largescale path loss is an exponential function of the separation distance between the Tx and Rx as expressed by

$$
P_{\mathrm{L}}(d)=P_{\mathrm{L}}\left(d_{0}\right)+10 n \log _{10}\left(d / d_{0}\right)+X
$$

where $P_{\mathrm{L}}(d)$ denotes the path loss in decibels at a distance $d$ between Tx and $\mathrm{Rx}, P_{\mathrm{L}}\left(d_{0}\right)$ is the path loss at a reference distance $d_{0}$, and $n$ is the path loss exponent. $X$ is a zero mean Gaussian random variable with standard deviation $\sigma$, which is equal to the standard deviation of the measurement samples from the estimated path loss curve. Using linear regression analysis, the path loss exponent $n$ that minimizes the difference between the measured and modeled values is determined.

\section{B. Tow-ray path loss model}

The two-ray path loss model considers not only the direct path but also a reflected one from the ground. This model is widely used in literature to model the LoS channel in V2X. The received power according to the two-ray path loss at Tx$\mathrm{Rx}$ separation distance $d$ is given by

$$
P_{\mathrm{L}}(d)=20 \log _{10}\left(\frac{4 \pi}{\lambda}\right)-20 \log _{10}\left|\frac{e^{-j k d_{\mathrm{LOS}}}}{d_{\mathrm{LOS}}}+\Gamma(\theta) \frac{e^{-j k d_{\mathrm{gr}}}}{d_{\mathrm{gr}}}\right|
$$

where the parameter $k=2 \pi / \lambda$ is the wavenumber at the center frequency $f_{\mathrm{c}}$ and $\lambda$ is the wavelength. $d_{\mathrm{LOS}}=$ $\sqrt{d^{2}+\left(h_{\mathrm{Tx}}-h_{\mathrm{Rx}}\right)^{2}}$ and $d_{\mathrm{gr}}=\sqrt{d^{2}+\left(h_{\mathrm{Tx}}+h_{\mathrm{Rx}}\right)^{2}}$ are the propagation distances for the LOS and the ground reflection. $h_{\mathrm{Tx}}$ and $h_{\mathrm{Rx}}$ are the heights of the Tx antenna and the Rx antenna respectively. $\Gamma(\theta)$ is the ground reflection coefficient for the reflection angle $\theta$ and is calculated for vertical polarization by

$$
\Gamma(\theta)=\frac{\epsilon_{\mathrm{r}} \sin (\theta)+\sqrt{\epsilon_{\mathrm{r}}-\cos ^{2}(\theta)}}{\epsilon_{\mathrm{r}} \sin (\theta)-\sqrt{\epsilon_{\mathrm{r}}-\cos ^{2}(\theta)}}
$$

where $\epsilon_{\mathrm{r}}$ is the relative permittivity of the ground and $\theta=$ $\tan ^{-1}\left(\frac{h_{\mathrm{Tx}}+h_{\mathrm{Rx}}}{d}\right)$.

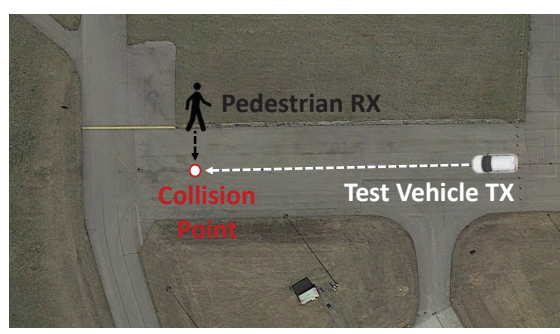

Fig. 1: Aerial view of the measurement scenario showing the trajectories of the TX and the RX towards the imaginary collision point. (Google Maps 2017 Geobasis-de/BKG.)

\section{MEASUREMENT CAMPAign}

To get more insight into the propagation of electromagnetic waves between vehicles and pedestrians, we performed a measurement campaign in March 2017 at the airport in Oberpfaffenhofen near Munich. This location was chosen since it represents a controlled environment with open-sky visibility and a small number of far-located objects that could potentially reflect or scatter the electromagnetic waves.

For our measurement campaign, a collision scenario of a vehicle and a pedestrian was considered. Here, the test vehicle drove straight towards the pedestrian approaching from the right side. Both the pedestrian and the vehicle met at an imaginary collision point. Fig. 1 displays the trajectory of both the test vehicle and the pedestrian on an aerial view of Google Maps. In our experiments, the test vehicle acted as the transmitter, while the pedestrian played the receiver role. The next two sections describe the test scenario and the measurement system.

\section{A. Measurement Scenario}

The test vehicle in which the transmitter was located was a Mercedes G400 (Fig. 2a). After an initial acceleration phase, the vehicle moved from $100 \mathrm{~m}$ distance towards the collision point with constant speed of $11 \mathrm{~m} / \mathrm{s}$. To study the influence of movement and body shadowing of the pedestrian, we performed tests with a static and with a moving pedestrian. In the static case, the pedestrian (Fig. 2b) was replaced by a tripod (Fig. 2c). The tripod was placed at three different positions with different distances $(12 \mathrm{~m}, 7 \mathrm{~m}$ and $2 \mathrm{~m})$ from the collision point. In the moving case, the pedestrian was approaching the collision point from $12 \mathrm{~m}$ distance at a speed of approximately $1.2 \mathrm{~m} / \mathrm{s}$. The antenna height for the test vehicle, the pedestrian and the tripod were $2 \mathrm{~m}, 1.3 \mathrm{~m}$ and $1.1 \mathrm{~m}$, respectively.

To study the impact of the parked vehicles at the roadside on the propagation channel especially the blockage of the LoS path, a row of cars and vans were parked in a line parallel to the trajectory of the test vehicle. Five different constellations using one to six vehicles of different size and shape were tested. Additionally, one of the cars was used to study the effect on the communication channel of an additional reflection coming from the opposite roadside, once, from a parked car and, once, from a moving car. Further, to study the effect of the shadowing of a crowd of people surrounding the pedestrian, the pedestrian was surrounded by four test persons. In addition, 


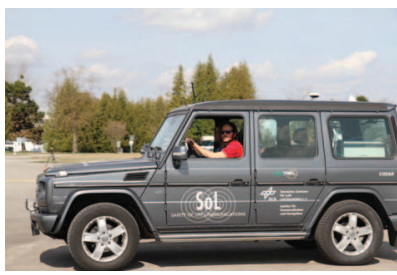

(a) TX Test Vehicle

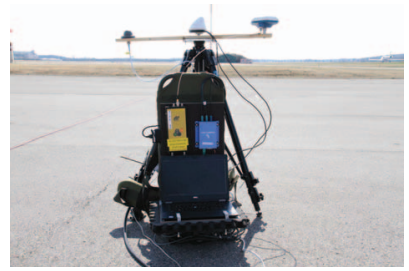

(c) RX Tripod

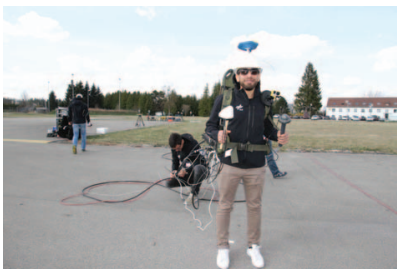

(b) RX Pedestrian

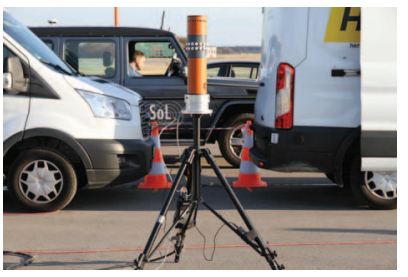

(d) RX Antenna Array
Fig. 2: Tranmitter (TX) and receiver (RX) involved in the measurement campaign.

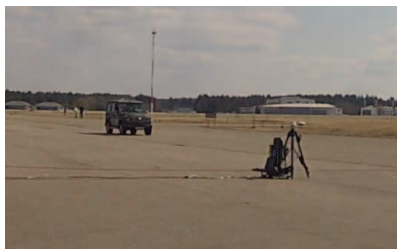

(a) LoS with tripod

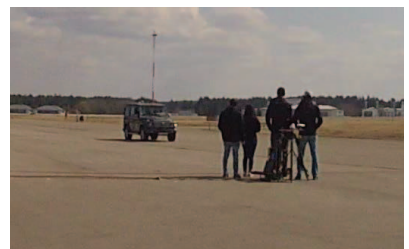

(c) Shadowing crowd

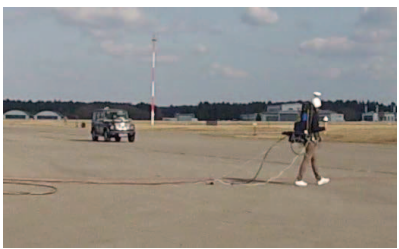

(b) LoS with moving pedestrian

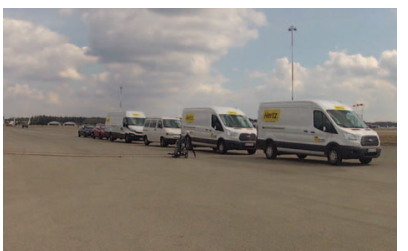

(d) Non-LoS with static tripod
Fig. 3: Four measurement scenarios addressed in this paper.

a circular antenna array with $2 \times 16$ elements was used ( See Fig. 2d) in order to detect the angle of arrival of different multipath components and study scattering phenomena.

All in all, a total set of 30 experiments with different combinations of moving/static pedestrian, LoS/non-LoS, different combinations of parked vehicles and with/without crowd shadowing were performed. In this paper, we will focus our attention on the following four scenarios:

1) LoS condition with static tripod as in Fig. $3 a$

2) LoS condition with moving pedestrian as in Fig. 3b

3) LoS condition with shadowing crowd as in Fig. 3c

4) Non-LoS condition with static tripod as in Fig. 3d

\section{B. Measurement Systems}

Both, the transmitter and the receiver, were equipped in parallel with two different measurement systems.
TABLE I: Channel sounder parameters

\begin{tabular}{ll}
\hline Parameter & Value \\
\hline RF center frequency & $5.2 \mathrm{GHz}$ \\
Bandwidth $B$ & $120 \mathrm{MHz}$ \\
Transmit power & $37 \mathrm{dBm}$ \\
Signal period $T_{\mathrm{p}}$ & $0.8 \mu \mathrm{s}$ \\
Time grid $T_{\mathrm{g}}$ & $1.024 \mathrm{~ms}$ \\
Tx antenna & Onmi-directional (V-polarized), $8 \mathrm{dBi}$ \\
Rx antenna & Omni-directional (V-polarized), $8 \mathrm{dBi}$ and \\
& Dual-polarized array \\
Vehicle speed & $11 \mathrm{~m} / \mathrm{s}$ \\
Pedestrian speed & $1.2 \mathrm{~m} / \mathrm{s}$ \\
\hline
\end{tabular}

1) ITS-G5: We performed measurements of the V2P channel in the $5.9 \mathrm{GHz}$ band. The vehicle, as a transmitter, and the pedestrian/tripod, as a receiver, were equipped each with a Cohda Wireless MK5 unit that incorporates dual 802.11p radios and Global Navigation Satellite System (GNSS) positioning system. A notebook was used for control and logging. The Cohda units were operating in the control channel number 180. Three modulation and coding schemes were used. Each modulation and coding scheme provides specific data rate and receiver sensitivity. The transmitter was configured to transmit at an update rate of $100 \mathrm{~Hz}$ for each modulation and coding scheme, and the packet length was set to be 400 Byte. Channel characterization based on this measurement system will be presented in future work.

2) Channel Sounder: The wideband channel sounding measurements were performed by using the RUSK-DLR channel sounder at center frequency $f_{\mathrm{c}}=5.2 \mathrm{GHz}$. The measurements bandwidth was $B=120 \mathrm{MHz}$, which corresponds to a delay resolution of $\Delta \tau=8.33 \mathrm{~ns}$. The channel impulse response was recorded every $T_{\mathrm{g}}=1.024 \mathrm{~ms}$, which allow to record a maximum absolute Doppler frequency of $f_{\mathrm{d}}=488 \mathrm{~Hz}$. To fulfill the requirement of maximum Doppler frequency, the vehicle velocity was restricted to $v_{\max }=11 \mathrm{~m} / \mathrm{s}$. The length of each channel impulse response (CIR) snapshot was $T_{\mathrm{p}}=0.8$ $\mu s$. The configuration parameters of the channel sounder are summarized in Table. I. The transmit antenna was positioned at the front side of the roof of the vehicle at a height of $h_{\mathrm{Tx}}=$ $1.9 \mathrm{~m}$ above the ground. The receive antenna was placed either on a tripod or carried by a pedestrian at heights of $h_{\mathrm{Rx}}=1.1 \mathrm{~m}$ or $1.3 \mathrm{~m}$.

In order to synchronize the transmitter and the receiver, two Rubidium clocks were used. However, during post processing, a clock drift had been recorded. To compensate the drift, the position was used to calculate the difference between the propagation delay of the LoS path in the CIR and the true propagation delay. The drift values were calculated in all experiments. It had been found that the drift increased linearly during the measurements day. Based on the linear increase in the drift, the value of the drift for each measurement snapshot were calculated and compensated. GNSS was used as a ground truth for the position of both transmit and receive antennas. The vehicle, the tripod and the pedestrian were equipped with a Topcon Legacy E+ L1/L2 GLONASS/GPS receiver. 
A geodetic-grade GNSS antenna was placed on the roof of the vehicle, on one end of the tripod and attached to the helmet of the pedestrian (see Fig. 2). The $10 \mathrm{~Hz}$ recorded GPS and GLONASS raw data were post processed to find a carrier-fixed solution with centimeter-level accuracy $(1 \sigma)$. The displacement between GNSS and the communication antennas at RX and TX was considered when computing the exact position for the propagation analysis with the channel sounder and the ITS-G5 system. To determine the location of the parked vehicles acting as obstruction to the LoS, an LDMRS multi-layer laser scanner from Sick was attached at the front bumper of the test vehicle and a ublox LEA 4T GPS receiver was employed. In post-processing, the laser point cloud was transformed from a vehicle coordinate frame to a global coordinate frame using the code-solution from the GPS receiver.

\section{DATA ANALYSis}

During the measurements, the CIR snapshots, measured periodically with the period $T_{\mathrm{g}}$, are denoted as $h\left(t_{k}, \tau_{q}\right)$, where $t_{k}=k \cdot t_{\mathrm{g}}$, with $k=0,1,2 \ldots$, being the time index of the measured CIR snapshot, and with $q=0, \ldots, N-1$, being the delay index of sample $q$, and $N$ is the number of samples in the CIR snapshot.

The power delay profile (PDP) is then calculated by

$$
P\left(t_{k}, \tau_{q}\right)=\left|h\left(t_{k}, \tau_{q}\right)\right|^{2} .
$$

Based on the PDP, the received power $P_{r}\left(t_{k}\right)$ can be calculated as

$$
P_{\mathrm{r}}\left(t_{k}\right)=\sum_{q=0}^{N-1} P\left(t_{k}, \tau_{q}\right) .
$$

Thereafter, the path loss $P_{\mathrm{L}}$ is obtained by

$$
P_{\mathrm{L}}=\frac{P_{\mathrm{t}} G_{\mathrm{Tx}} G_{\mathrm{Rx}}}{P_{\mathrm{r}}}
$$

where $P_{\mathrm{t}}, P_{\mathrm{r}}, G_{\mathrm{Tx}}$ and $G_{\mathrm{Rx}}$ are the power and the gain of the transmitter and the receiver antenna respectively.

\section{A. LoS scenario}

In Scenario 1, the receiver antenna is fixed on a static tripod at height $h_{\mathrm{Tx}}=1.1 \mathrm{~m}$. The tripod was $7 \mathrm{~m}$ away from the collision point (see Fig. 3a). Fig. 4 shows the obtained path loss versus the distance between the transmitter and receiver. As a comparison, the two-ray path loss model and the logdistance path loss model are also visualized. A typical tworay effect, i.e., the LoS path and the ground reflected path or multiple-ray, can be noticed, which causes a power variation slowly over the distance between the transmitter and receiver. With an estimated relative permittivity $\epsilon_{\mathrm{r}}=1.05$ for the ground and standard deviation $\sigma=3.20 \mathrm{~dB}$, the two-ray model fits the measurement data at large distance between the transmitter and receiver, i.e. $>40 \mathrm{~m}$, whereas for short distance the two-ray model does not fit well. One possible reason for the mismatch at distance smaller than $40 \mathrm{~m}$ could be that the LoS path is partially blocked and diffracted on the rooftop of the vehicle. The log-distance path loss model shown

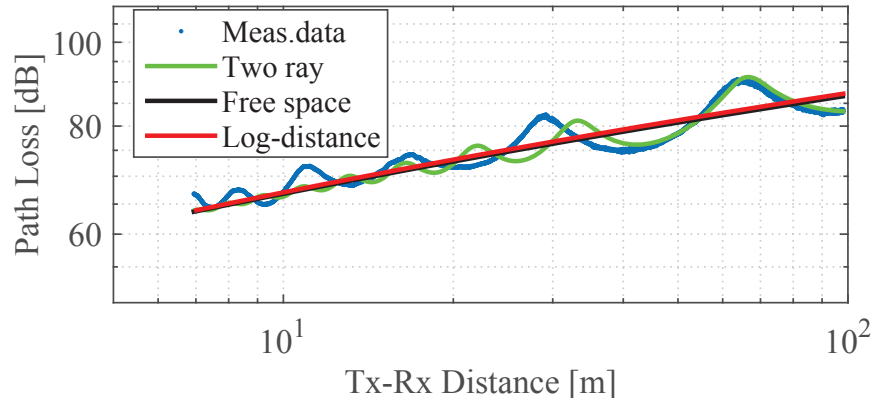

Fig. 4: Measured and modeled path loss for Scenario 1: LoS with static receiver/tripod.

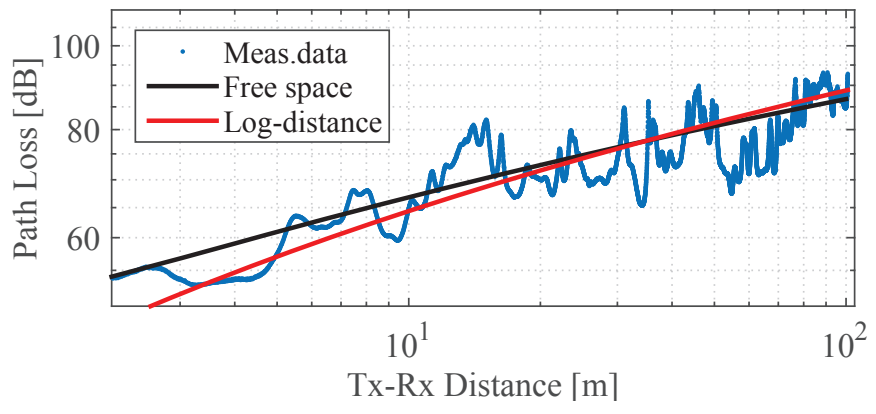

Fig. 5: Measured and modeled path loss for Scenario 2: LoS with moving receiver/pedestrian.

in Fig. 4 is estimated in a minimum mean square error sense with a reference distance $d_{0}=1 \mathrm{~m}$. Corresponding results are listed in Table. II.

In Scenario 2, the receive antenna was mounted with a moving pedestrian (see Fig. 3b). Fig. 5 shows the corresponding path loss versus the distance between the transmitter and receiver. As a comparison, the free space loss model and the log-distance model are also considered. The two-ray model does not fit to the measurement data and, therefore, is not visualized. A rapid fluctuation of the measured path loss can be seen, which maybe the result of the multipath component originated by the human body. Further, the moving human body also results in dynamic antenna attitude and position. The estimated path loss exponent $n$ is 2.44 that is larger than the $n$ for the free space propagation.

\section{B. Crowd shadowing scenario}

In Scenario 3 (see Fig. 3c) we evaluate the influence of neighboring pedestrians on the received power. Fig. 6 shows the obtained path loss from the measurement. Due to the shadowing effect caused by the crowd around the Rx, the two-

TABLE II: Log-distance path loss model parameters based on wideband measurement data.

\begin{tabular}{llll}
\hline Scenario & $n$ & $P_{\mathrm{L}}\left(d_{0}\right)[\mathrm{dB}]$ & $\sigma[\mathrm{dB}]$ \\
\hline 1 & 2.03 & 46.77 & 3.20 \\
\hline 2 & 2.44 & 40 & 5.47 \\
\hline 3 & 1.26 & 67 & 3.35 \\
\hline
\end{tabular}




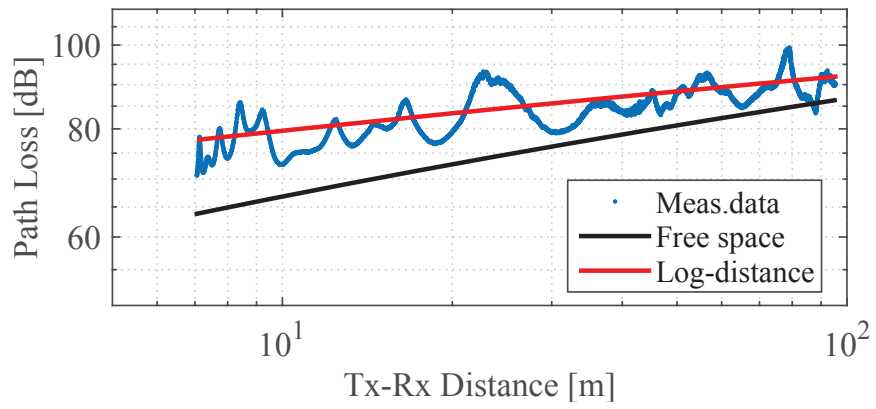

Fig. 6: Measured and modeled path loss for Scenario 3: crowd shadowing.

ray effect can not be clearly observed. In general, the measured path loss value is $5-10 \mathrm{~dB}$ larger than the LoS case. The estimated path loss exponent $n$ is 1.26 that is smaller than the $n$ in Scenario 1. It indicates that the power in Scenario 3 decreases slower than in Scenario 1, however, with more shadowing caused by the pedestrians, i.e. a higher value of $P_{\mathrm{L}}\left(d_{0}\right)=67 d B$. The similar finding is also reported in [10].

\section{NLoS scenario}

In Scenario 4, the receive antenna was stationary next to a convoy consisting of 6 vehicles with different sizes. Adjacent vehicles are separated with a gap of $1 \mathrm{~m}$ (see Fig. 3d). During the movement of the transmitter toward the receiver, the LoS is partially or completely obstructed. Fig. 7 shows the measurement result for the path loss values. The measurement samples displayed in blue between 67 and $100 \mathrm{~m}$ are obtained under LoS condition without obstruction by the parked vehicles. It can be seen that the path loss value is similar to the LoS condition in Fig. 4 between 50 and $100 \mathrm{~m}$. The measurement samples in yellow and in magenta correspond to the obstruction of the LoS by the black and red vehicles ( $1.5 \mathrm{~m}$ height), respectively. The average path loss value is $10 \mathrm{~dB}$ larger than the free space path loss. A rapid fluctuation of the path loss values can be noticed in terms of the samples in red and in black, where the LoS path is blocked by large size vehicles $(2.62 \mathrm{~m}$ height $)$. The smaller vehicle ( $1.98 \mathrm{~m}$ height $)$ corresponding to the green samples causes loss on average $15 \mathrm{~dB}$.

\section{CONCLUSION}

In this paper, we presented an extensive wideband measurement campaign for vehicle-to-pedestrian communications. The measurement setup and the scenarios were described in detail. First results of the path loss models for four different scenarios are presented based on the wideband channel measurements at $5.2 \mathrm{GHz}$. The two-ray path loss model fits the measured path loss at distances between $40 \mathrm{~m}$ and $100 \mathrm{~m}$ better than the log-distance path loss model. It has been found that the reflection of the signal by the pedestrian body causes rapid fluctuations of the path loss around its mean value. In addition, other pedestrians that surround the receiver cause path loss variations and degradation in the order of $5 \mathrm{~dB}$ to $10 \mathrm{~dB}$ due to shadowing. LoS obstruction by parked vehicles is investigated

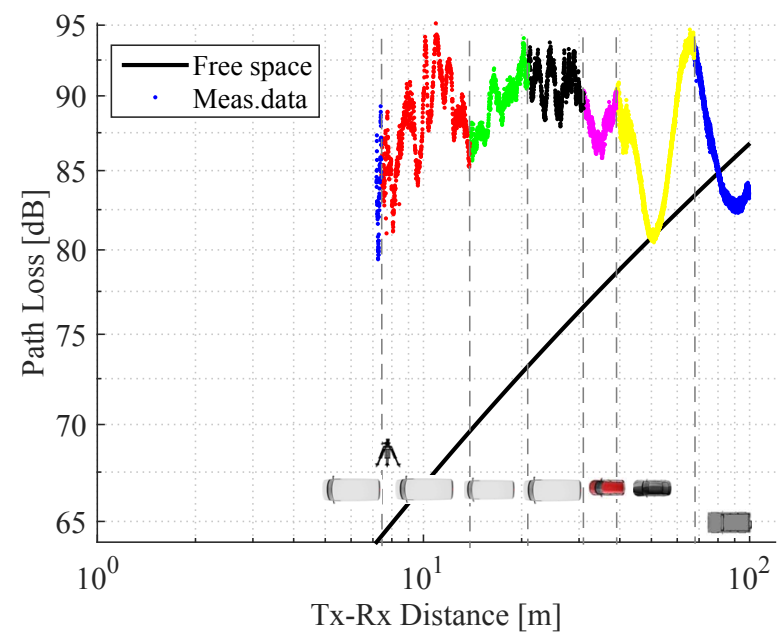

Fig. 7: Measured path loss for Scenario 4: NLoS with static receiver/tripod. Parked vehicles are shown to illustrate the cause of LoS obstruction in each zone.

and the extra path loss of about $10 \mathrm{~dB}$ to $15 \mathrm{~dB}$ which is caused by each individual parked vehicle is distinguished.

Future work will include enhancing the two-ray path loss model by including a diffraction model for distances below $40 \mathrm{~m}$. Moreover, it will include repeating the characterization using the data obtained using ITS-G5 system at $5.9 \mathrm{GHz}$.

\section{ACKNOWLEDGEMENT}

The authors would like to thank Michael Walter, Estefania Munoz Diaz-Ropero, Dina Bousdar Ahmed, Paul Unterhuber, Mohammad Soliman and Luis Enrique Dez Blanco for their assistance during the measurement campaign.

\section{REFERENCES}

[1] European Commission, "Road safety in the European Union - Trend, Statistics and main Challenges", March 2015

[2] F. de Ponte Müller, "Survey on Ranging Sensors and Cooperative Techniques for Relative Positioning of Vehicles" in Sensors, vol. $17 \mathrm{~m}$ no. 2, art. no. 271,2017

[3] I. Rashdan et al., "Performance Evaluation of Vehicle-to-Vehicle Communication for Cooperative Collision Avoidance at Urban Intersections," IEEE 86th Vehicular Technology Conference: 24-27 Sep 2017, Toronto, Canada.

[4] A.F. Molisch et al., "A survey on vehicle-to-vehicle propagation channels," IEEE Wireless Communications, 16(6), pp.12-22.

[5] W. Viriyasitavat et al., "Vehicular communications: Survey and challenges of channel and propagation models," IEEE Vehicular Technology Magazine, 10(2), pp.55-66, 2015.

[6] M.G. Doone, and S.L. Cotton, "First-order characteristics of the personto-vehicle channel at 5.8 GHz." In Antennas and Propagation (EuCAP), 2014 8th European Conference on, pp. 848-851. IEEE, 2014.

[7] Z. Liu et al., "Implementation and performance measurement of a V2X communication system for vehicle and pedestrian safety," International Journal of Distributed Sensor Networks 12, no. 9 (2016)

[8] Anaya, J.J. Anaya et al., "Vehicle to pedestrian communications for protection of vulnerable road users," In Intelligent Vehicles Symposium Proceedings, pp. 1037-1042. IEEE, 2014.

[9] M. Jutila et al., "ITS-G5 performance improvement and evaluation for vulnerable road user safety services," IET Intelligent Transport Systems, 2017.

[10] H. Fernndez et al., "Path loss characterization for vehicular communications at $700 \mathrm{MHz}$ and 5.9 GHz under LOS and NLOS conditions," IEEE Antennas and Wireless Propagation Letters, 13, pp.931-934, 2014. 